TRANSACTIONS OF THE

AMERICAN MATHEMATICAL SOCIETY

Volume 188, Issue 2, 1974

\title{
SPECTRA OF POLAR FACTORS OF HYPONORMAL OPERATORS(1)
}

BY

\author{
C. R. PUTNAM
}

ABSTRACT. An investigation is made of the interdependence and properties of the spectrum of a hyponormal operator $T$ and of the spectra, and absolutely continuous spectra, of the factors in a polar factorization of $T$ when the latter exists.

1. Introduction. Only bounded operators on a fixed separable Hilbert space $-H$ will be considered in this paper. An operator $T$ will be said to have a polar factorization if $T=U P$ where $U$ is unitary and $P$ is a nonnegative selfadjoint operator. (Other factorizations in which $U$ is not unitary but is only an isometry or a partial isometry, cf. Halmos [1, p. 68], or Kato [3, p. 334], will not be considered.) Thus, if $T$ has a polar factorization $T=U P$, then $T^{*}=P U^{*}$ and $T^{*} T$ $=P^{2}$, hence $P=\left(T^{*} T\right)^{1 / 2}$, so that

$$
T=U P, \quad U \text { unitary and } P=\left(T^{*} T\right)^{1 / 2} .
$$

In general the unitary factor is not unique. In case $T$ is nonsingular, that is, if $\mathbf{0}$ is not in its spectrum, the polar factorization exists, is unique, and was given by Wintner [12]; a generalization was obtained by von Neumann [4, p. 307].

As noted above, if $T=U P$ where $U$ is unitary and $P$ is nonnegative then necessarily $P=\left(T^{*} T\right)^{1 / 2}$. Also,

$$
\left.T T^{*}=U\left(T^{*} T\right) U^{*} \text { (equivalently, }\left(T T^{*}\right)^{1 / 2}=U\left(T^{*} T\right)^{1 / 2} U^{*}\right), U \text { unitary. }
$$

Conversely, it was show a by Hartman [2], using the above mentioned result of von Neumann, that if $T$ is arbitrary then the nonzero spectra of $T^{*} T$ and $T T^{*}$ are identical, including multiplicities of both point and continuous spectra, while 0 may occur in the point spectra of $T^{*} T$ and $T T^{*}$ with different multiplicities. Further, (1.2) holds for some unitary $U$ if and only if the multiplicities of 0 in the point spectra of $T^{*} T$ and $T T^{*}$ (equivalently, of $T$ and of $T^{*}$ ) are equal, that is,

$$
\operatorname{dim}\{x: T x=0\}=\operatorname{dim}\left\{x: T^{*} x=0\right\} .
$$

Received by the editors February 21, 1973.

AMS (MOS) subject classifications (1970). Primary 47B20, 47A10, 47B15; Secondary $47 \mathrm{~B} 47$. Key words and phrases. Hyponormal operators, polar factorization, spectra of operators, absolutely continuous spectra.

(1) This work was supported by a National Science Foundation research grant. 
In addition (cf. [2, p. 234], $T$ has a polar factorization (1.1), for some unitary $U$, if and only if (1.2) holds for some (not necessarily the same) unitary $U$, or, equivalently, if and only if (1.3) holds. In this case, the unitary operator $U$ of (1.1) (but, of course, not that of (1.2)) is uniquely determined if 0 is not in the point spectrum of $T$ (and/or $T^{*}$ ), that is, if the common dimension of (1.3) is 0 .

Next, an operator is said to be hyponormal if

$$
T^{*} T-T T^{*}=D \geq 0,
$$

and completely hyponormal if, in addition, there is no nontrivial subspace reducing $T$ on which $T$ is normal. It was shown in Putnam [8] that if $T$ is completely hyponormal then its spectrum, $\mathrm{sp}(T)$, has positive planar measure and, in fact,

if $T$ is completely hyponormal then meas $_{2}(\operatorname{sp}(T) \cap \alpha)>0$ whenever $\operatorname{sp}(T) \cap \alpha \neq$ empty set,

where $\alpha$ denotes any open disk of the complex plane.

Let $T_{z}=T-z I$ for any complex $z$. Then $T_{z}^{*} T_{z}-T_{z} T_{z}^{*}=T^{*} T-T T^{*}$ and hence

$$
\left\{x: T_{z^{x}}=0\right\} \subset\left\{x: T_{z}^{*} x=0\right\} \text { if } T \text { is hyponormal. }
$$

Hence, if $z$ is in the point spectrum of a hyponormal $T$ the corresponding eigenspace is a reducing space of $T$ on which it is normal. It is also clear that if $T$ is hyponormal and if 0 is not in the point spectrum of $T^{*}$ then $T$ has a (unique) polar factorization (1.1). Of course, if $T$ is normal, and whether or not 0 is in the point spectrum of $T^{*}$, equality holds in (1.6) for all $z$, in particular, for $z=$ 0 , and it follows that $T$ must have a (that is, at least one) polar factorization. Such a factorization is easily constructed, for instance, from the spectral resolution of the operator. The unilateral shift (cf. Halmos [1, p. 40]) is an example of a completely hyponormal operator which fails to have a polar factorization (1.1).

Recall that $A$ is a selfadjoint operator with the spectral resolution $A=$ $\int t d E_{t}$ then the set $H_{a}(A)$ of elements $x$ in $H$ for which $\left\|E_{t} x\right\|^{2}$ is a n absolutely continuous function of $t$ is a subspace of $H$ reducing $A$. The operator $A$ is said to be absolutely continuous if $H_{a}(A)=H$. Similar concepts can be defined for a unitary operator $U=\int_{0}^{2 \pi} e^{i t} d E_{t}$; cf. [6, p. 19].

If $T$ is hyponormal with the rectangular representation $T=A+i B(A, B$ selfadjoint) it was shown in Putnam [5] (cf. also [6, p. 46]) that, exactly as in the case when $T$ is normal, the spectra of $A$ and $B$ are precisely the projections, as real sets, of the spectrum of $T$ onto the real and imaginary axes. Further (cf. $[6$, pp. 42-43]), both $H_{a}(A)$ and $H_{a}(B)$ contain the least subspace of $H$ reducing $T$ and containing the range of $T^{*} T-T T^{*}$. In particular, if $T$ is completely hyponormal, $A$ and $B$ are absolutely continuous. This paper will deal with an analogous 
investigation of the spectrum of $T$ and of the spectra, and absolutely continuous spectra, of the components of a polar factorization of $T$, when the latter exists.

For use below, recall that a number $t$ is said to be in the essential spectrum of a selfadjoint operator $A$, essp $(A)$, if $t$ is either a limit point of $\operatorname{sp}(A)$ or is an eigenvalue of infinite multiplicity. The point spectrum of any operator $T$ will be denoted by $\operatorname{ptsp}(T)$.

\section{Theorem 1. Let $T$ be byponormal and let} $z \in$ boundary of $\operatorname{sp}(T)$.

Then

$$
|z| \epsilon \operatorname{sp}\left(T^{*} T\right)^{1 / 2} \cap \operatorname{sp}\left(T T^{*}\right)^{1 / 2} \text {. }
$$

Further, if $T$ is completely byponormal, then

$$
|z| \epsilon \operatorname{essp}\left(T^{*} T\right)^{1 / 2} \cap \operatorname{essp}\left(T T^{*}\right)^{1 / 2} \text {. }
$$

Proof. The hypothesis (2.1) implies that there exists a sequence of unit rectors, $\left\{x_{n}\right\}$, for which $(T-z I) x_{n} \rightarrow 0$. Since $T$ is hyponormal, also $\left(T^{*}-\bar{z} I\right) x_{n} \rightarrow$ 0 and so $\left(T^{*} T-|z|^{2} I\right) x_{n} \rightarrow 0$ and $\left(T T^{*}-|z|^{2} I\right) x_{n} \rightarrow 0$, hence also $\left(\left(T^{*} T\right)^{1 / 2}-|z| I\right) x_{n} \rightarrow 0$ and $\left(\left(T T^{*}\right)^{1 / 2}-|z| I\right) x_{n} \rightarrow 0$, and so (2.2) follows. Further, if $z$ is an isolated point of $\operatorname{sp}(T), T$ has a normal part with eigenvalue $z$ (cf. Stampfli [11, p. 473] or Putnam [8]). Hence, if $T$ is completely hyponormal, it follows from [7, Theorem 2 of p. 506], that the above sequence $\left\{x_{n}\right\}$ can be chosen so as to converge weakly to 0 , and hence (2.3) holds.

Remarks. The above argument shows that if $T$ is normal, then (2.2) holds, if, instead of (2.1), it is supposed only that

$$
z \in \operatorname{sp}(T) \text {. }
$$

In general, however, if $T$ is only hyponormal, condition (2.4) does not imply (2.2). One need only let $T$ denote the unilateral shift operator, so that on the $l^{2}$ sequence space $x=\left(x_{1}, x_{2}, \ldots\right), T x=\left(0, x_{1}, x_{2}, \ldots\right)$. Then $\operatorname{sp}(T)$ is the closed unit disk but $T^{*} T=1$ and $T T^{*}=\operatorname{diag}(0,1,1, \ldots)$.

As noted earlier, the unilateral shift fails to have a polar factorization (1.1). However, even if $T$ is hyponormal and nonsingular, in which case a polar factorization (1.1) is assured, still (2.4) does not imply (2.2). To see this, consider the doubly infinite nonnegative diagonal matrices

$$
A=\operatorname{diag}\left(\ldots, a_{-1}, a_{0}, a_{1}, \ldots\right) \text { and } B=\operatorname{diag}\left(\ldots, b_{-1}, b_{0}, b_{1}, \ldots\right)
$$

with $a_{i}=4$ for $i \geq 1, a_{i}=1$ for $i \leq 0, b_{i}=4$ for $i \geq 0, b_{i}=1$ for $i \leq-1$. Let $P$ denote the nonnegative square root of $B$ and put $T=U P$, where $U$ is the unitary 
bilateral shift on the sequence space of vectors $x=\left(\ldots, x_{-1}, x_{0}, x_{1}, \ldots\right)$, $\Sigma\left|x_{i}\right|^{2}<\infty$, defined by $(U x)_{n}=x_{n-1}(n=0, \pm 1, \pm 2, \ldots)$. Since $A=U B U^{*}$ then

$$
T^{*} T-T T^{*}=B-A=\operatorname{diag}\left(\ldots, d_{-1}, d_{0}, d_{1}, \ldots\right)
$$

with $d_{0}=3$ and $d_{i}=0$ for $i \neq 0$. Thus $T$ is hyponormal but not normal. Also, $\operatorname{sp}(T)=\{z: 1 \leq|z| \leq 2\}$, as can be deduced, for instance, from the results of this paper (cf. Theorems 8,9 below). However, $\operatorname{sp}\left(T^{*} T\right)^{1 / 2}=\operatorname{sp}\left(T T^{*}\right)^{1 / 2}=\{1,2\}$.

Theorem 2. Let $T$ be byponormal and suppose that $z \in \mathrm{sp}(T)$ and $\bar{z} \notin$ ptsp $\left(T^{*}\right)$. Then $|z| \epsilon \operatorname{essp}\left(T^{*} T\right)^{1 / 2} \cap \operatorname{essp}\left(T T^{*}\right)^{1 / 2}$.

Proof. Since $T$ is hyponormal, $T_{z}^{*} T_{z} \geq T_{z} T_{z}^{*}$, where $T_{z}=T-z I$, and so, since $z \in \mathrm{sp}(T), 0 \in \mathrm{sp}\left(T_{z} T_{z}^{*}\right)$. Also, since $\bar{z} \notin \mathrm{ptsp}\left(T^{*}\right)$, then $z \notin \mathrm{ptsp}(T)$. Consequently, 0 is in the essential spectra of both $T_{z}^{*} T_{z}$ and $T_{z} T_{z}^{*}$. In view of the inequality $T_{z}^{*} T_{z} \geq T_{z} T_{z}^{*}$, there exists a sequence of unit vectors, $\left\{x_{n}\right\}$, converging weakly to 0 for which both $(T-z I) x_{n} \rightarrow 0$ and $\left(T^{*}-\bar{z} I\right) x_{n} \rightarrow 0$ and hence $\left(T^{*} T-|z|^{2} I\right) x_{n} \rightarrow 0$ and $\left(T T^{*}-|z|^{2} I\right) x_{n} \rightarrow 0$. Thus, $|z|^{2}$ is in the essential spectra of both $T^{*} T$ and $T T^{*}$, and the assertion of the theorem follows.

3. Theorem 3. Let $T$ be byponormal with a polar factorization (1.1). Suppose that $z \neq 0$ and satisfies (2.4) and that $z=|z| e^{i \theta}$. Then, for any $U$ of (1.1),

$$
e^{i \theta} \in \operatorname{sp}(U) \text {. }
$$

Proof. Let $z_{1}=r e^{i \theta}$ where $r=\max \left\{|z|: z=|z| e^{i \theta}\right.$ and $\left.z \epsilon \mathrm{sp}(T)\right\}$ (hence $r>0)$. Clearly, $z_{1}$ is a boundary point of $\operatorname{sp}(T)$ and, as in Theorem 1 , there exists a seque nce of unit vectors, $\left\{x_{n}\right\}$, such that $\left(T-z_{1} I\right) x_{n} \rightarrow 0$ and $\left(T^{*}-\bar{z}_{1} I\right) x_{n}$ $\rightarrow 0$ and hence also $\left(\left(T^{*} T\right)^{1 / 2}-r I\right) x_{n} \rightarrow 0$. But $\left(T-z_{1} I\right) x_{n}=U\left(T^{*} T\right)^{1 / 2} x_{n}-z_{1} x_{n}$ $\rightarrow 0$. Since $r>0$, this implies that $\left(U x_{n}-e^{i \theta_{x_{n}}}\right) \rightarrow 0$ and, hence, that (3.1) holds.

4. Theorem 4. Let $T$ be byponormal and nonsingular, so that $T$ bas a (unique) polar factorization (1.1). Then if $e^{i \theta} \in \mathrm{sp}(U)$, there exists a $z=|z| e^{i \theta} \neq 0$ satisfying (2.4).

Proof. We have $T=U P$ and

$$
P^{2}-U P^{2} U^{*}=T^{*} T-T T^{*}=D \geq 0 .
$$

Since $e^{i \theta} \in \operatorname{sp}(U)$ there exists a sequence of unit vectors, $\left\{x_{n}\right\}$, satisfying $\left(U-e^{i \theta} I\right) x_{n} \rightarrow 0$, hence $\left(U^{*}-e^{-i \theta} I\right) x_{n} \rightarrow 0$. Clearly,

$$
\begin{aligned}
\left\|D^{1 / 2} x_{n}\right\|^{2} & =\left(D x_{n}, x_{n}\right)=\left(P^{2} x_{n}, x_{n}\right)-\left(U P^{2} U^{*} x_{n}, x_{n}\right) \\
& =\left(P^{2} x_{n}, x_{n}\right)-\left(P^{2} U^{*} x_{n}, U^{*} x_{n}\right) \rightarrow 0
\end{aligned}
$$


and so $D x_{n} \rightarrow 0$. Hence, by (4.1); $P^{2} x_{n}-U P^{2} U^{*} x_{n} \rightarrow 0$, that is, $\left(U^{*}-e^{-i \theta} I\right) P^{2} x_{n}$ $\rightarrow 0$. A similar argument shows that $\left(U^{*}-e^{-i \theta} I\right) f^{n}\left(P^{2}\right) x_{n} \rightarrow 0$, where $f(t)$ is a polynomial, or, via the functional calculus, a continuous function on $(-\infty, \infty)$. It then follows (cf. a similar argument in $[6, \mathrm{p} .46]$ ) that there exists a number $s>0$ and a sequence of unit vectors $\left\{y_{n}\right\}$ such that $\left(P^{2}-s I\right) y_{n} \rightarrow 0$ and $\left(U^{*}-e^{\left.-i \theta_{l}\right) y_{n}}\right.$ $\rightarrow 0$, hence also $\left(P-s^{1 / 2} I\right) y_{n} \rightarrow 0$ and $\left(U-e^{i \theta} I\right) y_{n} \rightarrow 0$. Consequently, if $z=s^{1 / z} e^{i \theta}$, then $(T-z I) y_{n} \rightarrow 0$ (also $\left(T^{*}-\bar{z} I\right) y_{n} \rightarrow 0$ ) and so (2.4) holds.

5. Theorem 5. Let $T$ be byponormal with a polar factorization (1.1) and suppose that

$$
0 \notin \operatorname{ptsp}(T) \text {. }
$$

If $e^{i \theta} \in \mathrm{sp}(U)$ then there exist $z_{n}=\left|z_{n}\right| e^{i \theta_{n}} \neq 0, z \in \operatorname{sp}(T)$, for wbicb $\theta_{n} \rightarrow \theta$.

Remark. Note that if $T$ is completely hyponormal then the hypotheses (1.1) and (5.1) are certainly fulfilled.

Proof. In case $T$ is nonsingular the above theorem follows from Theorem 4. The theorem also is clear if $T$ is singular and if there does not exist some open wedge

$$
W=\left\{z: z=r e^{i t}, r>0, a<t<b\right\}, \quad a<\theta<b,
$$

for which

$$
\operatorname{sp}(T) \cap \|^{\prime} \text { is empty. }
$$

Consequently, it is sufficient to show that if $T$ is singular then the assumption that there exists a wedge $W$ of (5.2) satisfying (5.3) leads to a contradiction.

Suppose then the existence of such a wedge. Consider the bisector of $W$, that is, the half-line $\left\{z: z=r e^{i \frac{1}{2}(a+b)}, r>0\right\}$ and choose complex numbers $s_{n}=$ $\left|s_{n}\right| e^{i \frac{1}{2}(a+b)} \neq 0$ on this half-line satisfying $s_{n} \rightarrow 0$. It is clear that each (hyponormal) operator $T_{n}=T-s_{n} l$ is nonsingular and that, by (5.3), $\operatorname{sp}\left(T_{n}\right) \cap W$ is empty. If $T_{n}=U_{n} P_{n}$ is the (unique) polar factorization of $T_{n}$ then, by Theorem 4,

$$
e^{i t} \in \operatorname{sp}\left(U_{n}\right) \quad(n=1,2, \ldots) \text { whenever } a<t<b .
$$

But $\left\|T-T_{n}\right\| \rightarrow 0$ and hence $\left\|P-P_{n}\right\|=\left\|\left(T^{*} T\right)^{1 / 2}-\left(T_{n}^{*} T_{n}\right)^{1 / 2}\right\| \rightarrow 0$. Also, $U_{n} P-U P=T_{n}-T+U_{n}\left(P-P_{n}\right)$, so that $\left\|U_{n} P-U P\right\| \stackrel{n}{\rightarrow} 0$ and, in particular, $U_{n} P x \rightarrow U P x$ (strongly) for all $x$ in $H$. In view of (5.1), $0 \notin$ point spectrum of $P^{n}=\left(T^{*} T\right)^{1 / 2}$, hence the range of $P$ is dense, and consequently

$$
U_{n} \rightarrow U \text { (strongly). }
$$


By (5.4), $\left\|\left(U_{n}-e^{i \theta} I\right) x\right\| \geq c\|x\|$ for all $x$ where $c$ is some positive constant, and hence, by (5.5), $\left\|\left(U-e^{i \theta} I\right) x\right\| \geq c\|x\|$. This implies that $e^{i \theta} \notin \mathrm{sp}(U)$, a contradiction, and the proof of Theorem 5 is complete.

6. Theorem 6. Let $T$ be completely byponormal and have a polar factorization $T=U P$ of (1.1). In addition, suppose that there exists some open wedge W of (5.2) satisfying (5.3). Then

$P=\left(T^{*} T\right)^{1 / 2}$ (bence also $\left.\left(T T^{*}\right)^{1 / 2}\right)$ and $U$ are absolutely continuous.

Proof. It follows from Theorem 4 that no $e^{i \theta}, a<\theta<b$, can belong to $\operatorname{sp}(U)$. It now follows from (4.1) and the theorem of $[6, \mathrm{p} .21]$, that both $H_{a}\left(P^{2}\right)$ $\left(=H_{a}\left(T^{*} T\right)=H_{a}\left(\left(T^{*} T\right)^{1 / 2}\right)\right)$ and $H_{a}(U)$ contain the least subspace, $M$, of $H$ reducing $P^{2}$ and $U$ (equivalently, reducing $P$ and $U$ ) and containing the range of $D=$ $T^{*} T-T T^{*}$. Since $T$ is completely hyponormal, $M=H$, and, in particular, (6.1) follows.

\section{Theorem 7. Let $T$ be byponormal and suppose that}

$$
r \in \operatorname{sp}\left(T^{*} T\right) \quad(\text { bence } r \geq 0) \text {. }
$$

Then there exists a $z \in \mathrm{sp}(T)$ for which $|z|=r^{1 / 2}$.

Remark. The hypothesis (7.1) of Theorem 7 can be replaced by

$$
\left.r \in \operatorname{sp}\left(T T^{*}\right) \quad \text { (hence } r \geq 0\right) \text {. }
$$

In fact, if $r=0$ then $T$ is singular and $0 \in \mathrm{sp}(T)$. If $r>0$, then (7.1) implies (7.1); cf. $\$ 1$ above.

Proof. We first establish the theorem under the added hypothesis that $T$ has a polar factorization (1.1) and that $\operatorname{sp}(U)$ is not the entire circle $|z|=1$. Thus,

$$
T=U P \text { and } \operatorname{meas}_{1}(\operatorname{sp}(U))<2 \pi \text {. }
$$

Let $e^{i \theta} \notin \mathrm{sp}(U)$ and define the unitary operator $U_{\theta}=e^{-i \theta} U$. Then $1 \notin \mathrm{sp}\left(U_{\theta}\right)$ and relation (4.1) becomes $P^{2}-U_{\theta} P^{2} U_{\theta}^{*}=D$. Now, $U_{\theta}$ is the Cayley transform of a selfadjoint operator $A$, where

$$
U_{\theta}=(A-i I)(A+i I)^{-1} \quad\left(U_{\theta}=e^{-i \theta} U\right) .
$$

If $C=1 / 2(A+i I) D(A+i I)^{*}$, it is seen that

$$
A P^{2}-P^{2} A=i C, \quad C \geq 0 .
$$

(For a similar argument, see $[6$, pp. 16, 21].)

Next, by (7.1), $r \in \mathrm{sp}\left(P^{2}\right)$, so that $\left(P^{2}-r I\right) x_{n} \rightarrow 0$ for some sequence of

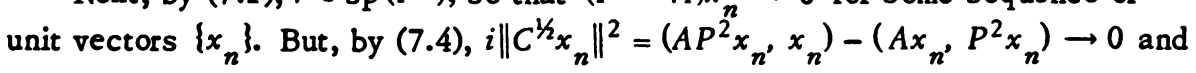


so $C x_{n} \rightarrow 0$, and hence by (7.4) again, $\left(P^{2}-r I\right) A x_{n} \rightarrow 0$. Similarly, one obtains

$$
\left(P^{2}-r I\right) A^{k} x_{n} \rightarrow 0 \text { as } n \rightarrow \infty(k=0,1,2, \ldots)
$$

In view of (7.3) and the relation $U_{\theta}^{*}=(A-i I)^{-1}(A+i I)$, it follows from (7.5) that

$$
\left(P^{2}-r I\right) U^{k} x_{n} \rightarrow 0, \quad n \rightarrow \infty(k=0, \pm 1, \pm 2, \cdots) .
$$

Hence, by an argument similar to that of $[6$, p. 46] (see also $\$ 4$ above) there exists some $e^{i \phi} \in \operatorname{sp}(U)$ and a sequence of unit vectors, $\left\{y_{n}\right\}$, such that $\left(P^{2}-r l\right) y_{n} \rightarrow 0$ (hence $\left.\left(P-r^{1 / 2} I\right) y_{n} \rightarrow 0\right)$ and $\left(U-e^{i \phi} I\right) y_{n} \rightarrow 0$. Thus, if $z=$ $r^{1 / 2} e^{i \phi}$ then $(T-z I) y_{n} \rightarrow 0$, thus $z \in \mathrm{sp}(T)$, and so Theorem 7 is proved in the special case in which (7.2) is assumed.

Next, we consider the general case of Theorem 7. Let $T$ have the rectangular representation $T=A+i B$ where $A$ has the spectral resolution $A=\int t d E_{t}$. Let $S_{n}=(-\infty, \infty)-(-1 / n, 1 / n)$ for $n=1,2, \ldots$ and consider the hyponormal operator $T_{n}=E\left(S_{n}\right) T E\left(S_{n}\right)$ defined on the Hilbert space $E\left(S_{n}\right) H$. Then $\operatorname{sp}\left(T_{n}\right)$ lies outside the strip $|\operatorname{Re}(z)|<1 / n$ (see [6, p. 46]) and also (see [8], [9])

$$
\operatorname{sp}\left(T_{n}\right) \subset \operatorname{sp}(T) .
$$

In particular, each $T_{n}$ is nonsingular and hence has a polar factorization $T_{n}=$ $U_{n} P_{n}$ and, by Theorem 4, meas $1\left(\operatorname{sp}\left(U_{n}\right)\right)<2 \pi(n=1,2, \cdots)$, so that (7.2) holds with the role of $T$ played by $T_{n}$. In addition, it is clear that

(7.8) $\quad T_{n} \rightarrow T, \quad T_{n}^{*} \rightarrow T^{*}$ (strongly) $\left[T_{n}\right.$ here as an operator on $H$ ].

Consequently, $T_{n}^{*} T_{n} \rightarrow T^{*} T$ (strongly) and, by (7.1), there exist $r_{n} \in \operatorname{sp}\left(T_{n}^{*} T_{n}\right)$ for which $r_{n} \rightarrow r$. (The argument is similar to that following formula line (5.5) above.) Since the assertion of Theorem 7 has already been proved for the $T_{n}$, there exist $z_{n} \in \operatorname{sp}\left(T_{n}\right)$ such that $\left|z_{n}\right|=r_{n}^{1 / 2} \rightarrow r^{1 / 2}$. Since, by (7.7), $z_{n} \in \mathrm{sp}(T)$, and since $\left\{z_{n}\right\}$ is a bounded sequence, there exists a convergent subsequence $\left\{z_{n_{k}}\right\}$, say $z_{n_{k}} \rightarrow z$. Clearly, this $z$ satisfies the conditions of Theorem 7 and the proof is complete.

8. Theorem 8. Let $T$ be completely byponormal and suppose that

$$
r(\geq 0) \text { is an isolated point of } \mathrm{sp}\left(T T^{*}\right) \text {. }
$$

Let $a=\inf \left(s: s \in \operatorname{sp}\left(T T^{*}\right), s \leq r\right.$ and, if $s<r,(s, r)$ contains no points of the essential spectrum of $\left.T T^{*}\right\}$; and $b=\sup \left\{s: s \in \operatorname{sp}\left(T T^{*}\right), s \geq r\right.$ and if $s>r,(r, s)$ contains no points of the essential spectrum of $\left.T T^{*}\right\}$. Then $a<b$ and either

$$
a<r \text { and }\left\{z: a^{1 / 2}<|z|<r^{1 / 2}\right\} \subset \operatorname{ptsp}\left(T^{*}\right)
$$

or 


$$
b>r \text { and }\left\{z: r^{1 / 2}<|z|<b^{1 / 2}\right\} \subset \operatorname{ptsp}\left(T^{*}\right)
$$

Remark. It should be noted that even if both inequalities $a<r<b$ hold, still, as simple examples show, only one of the relations (8.2) and (8.3) need hold.

Proof. First we show that $a<b$. Otherwise, $a=b=r$ and $\operatorname{sp}\left(T T^{*}\right)$ is the singleton $\{r\}$. If $r=0$ then $T=0$, hence $T$ is normal, a contradiction. If $r>0$ then, since $T^{*} T \geq T T^{*}, \operatorname{sp}\left(T^{*} T\right)=\operatorname{sp}\left(T T^{*}\right)=\{r\}$, that is, $T^{*} T=T T^{*}=r l$ and again $T$ must be normal, a contradiction.

It follows from Theorem 7 and the remark following it that there exists a number $z_{0} \in \operatorname{sp}(T)$ for which $\left|z_{0}\right|=r^{1 / 2}$. Also, there exist $z_{n} \in \operatorname{sp}(T)$ with $\left|z_{n}\right| \neq$ $\left|z_{0}\right|$ satisfying $z_{n} \rightarrow z_{0}$ as $n \rightarrow \infty$. Otherwise, there exists an open disk a centered at $z_{0}$ and such that $a \cap \mathrm{sp}(T)$ is not empty and has zero planar measure. This is impossible by (1.5). It follows from Theorem 1 and the definitions of $a$ and $b$ in Theorem 8 that no boundary points of $\operatorname{sp}(T)$ can lie in the difference set $\left\{z: a^{1 / 2}<|z|<b^{1 / 2}\right\}-\left\{z:|z|=r^{1 / 2}\right\}$.

Since $\left|z_{n}\right| \neq\left|z_{0}\right|=r^{1 / 2}$ then, for any $n$, either $\left|z_{n}\right|<r^{1 / 2}$ or $\left|z_{n}\right|>r^{1 / 2}$. Suppose first that $\left|z_{n}\right|<r^{1 / 2}$ for some $n$. Then clearly $a<r$ and, since no boundary point of $\operatorname{sp}(T)$ can lie in $\left\{z: a^{1 / 2}<|z|<r^{1 / 2}\right\}$, it follows that $\left\{z: a^{1 / 2}<|z|<r^{1 / 2}\right\} C$ $\operatorname{sp}(T)$. Relation (8.2) now follows from Theorem 2. Similarly, if $\left|z_{n}\right|>r^{1 / 2}$ for some $n$, relation (8.3) holds.

\section{Theorem 9. Let $T$ be completely byponormal and suppose that}

$$
\operatorname{meas}_{1}\left(\operatorname{sp}\left(T^{*} T\right)\right) \quad\left(=\operatorname{meas}_{1}\left(\operatorname{sp}\left(T T^{*}\right)\right)\right)=0 .
$$

Then there exists a finite or denumerably infinite number of pairwise disjoint open annuli $A_{n}=\left\{z: a_{n}<|z|<b_{n}\right\}(n=1,2, \ldots)$ sucb that

$$
\mathrm{sp}_{\mathrm{p}}(T) \text { is the closure of the set } \cup A_{n}
$$

and

$$
\bigcup A_{n} \subset \operatorname{ptsp}\left(T^{*}\right)
$$

Proof. Let $z_{0} \in \operatorname{sp}(T)$. Then consider any open disk $\alpha$ containing $z_{0}$. Then necessarily a contains a closed disk $\beta$ satisfying

$$
\beta=\left\{z:\left|z-z_{1}\right| \leq s, s>0\right\} \subset \operatorname{sp}(T)
$$

In fact, otherwise, all points of $a \cap \mathrm{sp}(T)$ would be boundary points of $\operatorname{sp}(T)$. Further, if the half-line $L: \theta=c$ (const.) intersects $\alpha$, then, by Theorem 1 , each $r \geq 0$ satisfying $r e^{i c} \in L \cap(a \cap s p(T))$ belongs to $\operatorname{sp}\left(T^{*} T\right)^{1 / 2}$. Hence, by (9.1), the set of such numbers $r$ has linear measure 0 . It readily follows from Fubini's theorem that $a \cap \operatorname{sp}(T)$ (which contains $z_{0}$ and hence is not empty) has zero 
planar measure and hence, by (1.5), $T$ is not completely hyponormal, a contradiction. This proves (9.4).

If $a=\inf \{|z|: z \in \beta\}$ and $b=\sup \{|z|: z \in \beta\}$ then $a<b$. By $(9.1), \operatorname{sp}\left(T^{*} T\right)^{1 / 2}$ cannot contain $[a, b]$ and it follows from Theorem 1 that

$$
\{z: a \leq|z| \leq b\} \subset \operatorname{sp}(T) \text {. }
$$

It is clear then that $\mathrm{sp}(T)$ is the closure of a set consisting of a possibly uncountable number of closed annuli each of the form (9.5). By a standard procedure (of combining intersecting annuli), one easily shows that $\mathrm{sp}(T)$ can be taken as the closure of a countable union of disjoint closed annuli, each of the form $\{z: c \leq|z| \leq d\}$ with $c<d$.

For a fixed such annulus let $U\left(c_{n^{\prime}} d_{n}\right)$ denote the canonical decomposition of the linear open set $[c, d]-\left\{[c, d] \cap \operatorname{sp}\left(T T^{*}\right)^{1 / 2}\right\}$. (Note that any $z$ satisfying $|z|=d$ is a boundary point of $\operatorname{sp}(T)$ and that a similar statement holds for $|z|=$ $c$ provided $c>0$. In view of $T^{*} T \geq T T^{*}$ it is clear from Theorem 1 that both $c$ and $d$ (even if $c=0$ ) belong to $\operatorname{sp}\left(T T^{*}\right)^{1 / 2}$.) It follows from (9.1) that $\{z: c \leq|z|$ $\leq d\}$ is the closure of $\bigcup_{B_{n}}$, where $B_{n}=\left\{z: c_{n}<|z|<d_{n}\right\}$, and, from Theorem 2, that each $B_{n} \subset$ ptsp $\left(T^{*}\right)$. This completes the proof of Theorem 9.

A final result is the following

Theorem 10. Let $T$ be byponormal and suppose that

$$
\operatorname{sp}\left(T T^{*}\right) \neq \text { interval. }
$$

Tben $T$ bas a nontrivial invariant subspace.

Proof. Clearly, it can be supposed that $T$ is completely hyponormal. Further, by (9.6) (cf. the beginning of the proof of Theorem 8), sp $\left(T T^{*}\right)$ contains at least two points $r_{1}$ and $r_{2}$ satisfying $r_{1}<r_{2}$ and for which $\left(r_{1}, r_{2}\right) \cap \operatorname{sp}\left(T T^{*}\right)$ is empty. It follows from Theorem 7 and the remark following it that there exist $z_{1}, z_{2} \in \operatorname{sp}(T)$ where $\left|z_{1}\right|=r_{1}^{1 / 2}$ and $\left|z_{2}\right|=r_{2}^{1 / 2}$.

Clearly, $T$ has a nontrivial invariant subspace if $\operatorname{ptsp}\left(T^{*}\right)$ is not empty. Hence, it can be supposed that this set is empty, and so, by Theorem $2, \operatorname{sp}(T)$ $\cap\left\{z: r_{1}^{1 / 2}<|z|<r_{2}^{1 / 3}\right\}$ is empty. Thus, sp $(T)$ is not connected and hence (cf. [10, p. 421]) $T$ has a nontrivial invariant subspace.

It may be noted that Theorem 10 is applicable to the unilateral shift but that it would not be if $(9.6)$ is replaced by the (stronger) condition $\operatorname{sp}\left(T^{*} T\right) \neq$ interval.

\section{REFERENCES}

1. P. R. Halmos, A Hilbert space problem book, Van Nostrand, Princeton, N. J., 1967. MR 34 \#8178.

2. P. Hartman, On the essential spectra of symmetric operators in Hilbert space, 
Amer. J. Math. 75 (1953), 229-240. MR 15, 136.

3. T. Kato, Perturbation theory for linear operators, Die Grundlehren der math. Wissenschaften, Band 132, Springer-Verlag, New York, 1966. MR 34 \#3324.

4. J. von Neumann, Über adjungierte Funktionaloperatoren, Ann. of Math. 33 (1932), 294-310.

5. C. R. Putnam, On the spectra of semi-normal operators, Trans. Amer. Math. Soc. 119 (1965), 509-523. MR $32 \# 2913$.

6. - Commutation properties of Hilbert space operators and related topics, Ergebnisse der Math. und ihrer Grenzgebiete, Band 36, Springer-Verlag, New York, 1967. MR 36 \#707.

7. The spectra of operators having resolvents of first order growth, Trans. Amer. Math. Soc. 133 (1968), 505-510. MR 37 \#4651.

8. - An inequality for the area of hyponormal spectra, Math. Z. 116 (1970), 323-330. MR $42 \# 5085$.

9. - A similarity between hyponormal and normal spectra, Illinois J. Math. 16 (1972), 695-702.

10. F. Riesz and B. Sz-Nagy, Leçons d'analyse fonctionnelle, 2nd ed., Akad. Kiadó, Budapest, 1953; English transl., Functional analysis, Ungar, New York, 1955. MR 15, $132 ; 17,175$.

11. J. G. Stampfli, Hyponormal operators and spectral density, Trans. Amer. Math. Soc. 117 (1965), 469-476. MR 30 \#3375.

12. A. Wintner, On non-singular bounded matrices, Amer. J. Math. 54 (1932), 145-149.

DEPARTMENT OF MATHEMATICS, PURDUE UNIVERSITY, WEST LAFAYETTE, INDIANA 47907 